\title{
Long-term outcome of vertebral artery origin stenosis in patients with acute ischemic stroke
}

\author{
Young Jin Kim ${ }^{1 \dagger}$, Joon Hwa Lee ${ }^{1 \dagger}$, Jin Woo Choi ${ }^{2}$, Hong Gee Roh²${ }^{2}$ Young II Chun³ ${ }^{3}$ Ji-Sung Lee ${ }^{4}$ \\ and Hahn Young Kim ${ }^{1 *}$
}

\begin{abstract}
Background: Vertebral artery origin (VAO) stenosis is occasionally observed in patients who have acute ischemic stroke. We investigated the long-term outcomes and clinical significance of VAO stenosis in patients with acute ischemic stroke.

Methods: We performed a prospective observational study using a single stroke center registry to investigate the risk of recurrent stroke and vascular outcomes in patients with acute ischemic stroke and VAO stenosis. To relate the clinical significance of VAO stenosis to the vascular territory of the index stroke, patients were classified into an asymptomatic VAO stenosis group and a symptomatic VAO stenosis group.

Results: Of the 774 patients who had acute ischemic stroke, 149 (19.3\%) of them had more than $50 \%$ stenosis of the VAO. During 309 patient-years of follow-up (mean, 2.3 years), there were 7 ischemic strokes, 6 hemorrhagic strokes, and 2 unknown strokes. The annual event rates were $0.97 \%$ for posterior circulation ischemic stroke, $4.86 \%$ for all stroke, and $6.80 \%$ for the composite cardiovascular outcome. The annual event rate for ischemic stroke in the posterior circulation was significantly higher in patients who had symptomatic VAO stenosis than in patients who had asymptomatic stenosis (1.88\% vs. $0 \%, \mathrm{p}=0.046)$. In a multivariate analysis, the hazard ratio, per one point increase of the Essen Stroke Risk Score (ESRS) for the composite cardiovascular outcome, was 1.46 (95\% Cl, 1.02-2.08, $\mathrm{p}=0.036)$.

Conclusions: Long-term outcomes of more than $50 \%$ stenosis of the VAO in patients with acute ischemic stroke were generally favorable. Additionally, ESRS was a predictor for the composite cardiovascular outcome. Asymptomatic VAO stenosis may not be a specific risk factor for recurrent ischemic stroke in the posterior circulation. However, VAO stenosis may require more clinical attention as a potential source of recurrent stroke when VAO stenosis is observed in patients who have concurrent ischemic stroke in the posterior circulation.
\end{abstract}

\section{Background}

A recent systematic review revealed that the recurrence rates of stroke and vascular events in medical secondary stroke prevention trials have declined substantially over the last 5 decades [1]. For recurrent stroke, the annual rate fell from $8.7 \%$ in trials that began in the 1960 s to $4.9 \%$ in trials that began in the 2000s [1].

Atherosclerosis of the vertebrobasilar artery is one of the major causes of ischemic stroke in the posterior circulation $[2,3]$. The most proximal portion of the extracranial vertebral artery is the second most common area of

\footnotetext{
* Correspondence: hykimmd@gmail.com

${ }^{\dagger}$ Equal contributors

'Department of Neurology, Konkuk University Medical Center, Research Institute of Medical Science, Konkuk University School of Medicine, Seoul, Republic of Korea

Full list of author information is available at the end of the article
}

stenosis, next to the carotid bifurcation [4]. In clinical practice, stenosis of vertebral artery origin (VAO) is occasionally observed in patients who have acute ischemic stroke. In a hospital-based cohort that consisted of patients who had various atherosclerotic arterial diseases, the risk of ischemic stroke in the posterior circulation was higher in patients who had VAO stenosis than in those who did not have VAO stenosis (annual rate, $0.4 \%$ vs. $0.1 \%$ ), even though the annual rate was very low [5]. However, VAO stenosis has not been well studied as a risk factor for stroke recurrence in patients with acute ischemic stroke, especially for posterior circulation ischemic stroke. Previous case series studies have reported controversial results. Stenosis of VAO could be an important embolic source for ischemic stroke in the posterior circulation $[3,6-9]$ or could be a benign condition because there is a 
good collateral supply from the contralateral vertebral artery or branches of the external carotid artery [10,11]. A recent pooled analysis study indicated that symptomatic vertebrobasilar stenosis can be a strong predictor of stroke recurrence [12]. Our study was performed to investigate the long-term outcomes in patients who had acute ischemic stroke and VAO stenosis and to examine the clinical significance of VAO stenosis as a potential risk factor for ischemic stroke in the posterior circulation or other cardiovascular outcomes.

\section{Methods}

\section{Patient inclusion and clinical assessments}

The subjects used in this study were a subset of patients enrolled in the Konkuk Stroke Registry, a single-center prospective observational cohort study that has been described elsewhere $[13,14]$. We evaluated the risk of recurrent stroke and vascular outcomes in patients with acute ischemic stroke and VAO stenosis who were recruited consecutively from December 2007 to December 2010. Acute ischemic stroke was confirmed by diffusionweighted MRI (DWI), which was performed within 5 days from the onset of stroke in patients who had been admitted consecutively to our center within $72 \mathrm{~h}$ after their stroke-onset. Based on the contrast-enhanced MR angiography (CE-MRA) data, trained neurologists (YJ Kim or JH Lee) determined the degree of VAO stenosis through a modified method according to the North American Symptomatic Carotid Endarterectomy Trial [15]. Patients who had more than 50\% stenosis of the VAO on CE-MRA images were recruited for the study. In cases of discrepancy, a senior stroke neurologist (HY Kim) made the final decision. Patients who underwent angioplasty and stenting for VAO stenosis in the acute period of the index stroke were excluded from the study. These exclusions were made because the purpose of our study was to investigate the long-term outcomes under optimal medical treatment.

Vascular risk factors, which included hypertension, diabetes mellitus, hyperlipidemia, cardiac arrhythmia, smoking, peripheral arterial disease, and previous stroke history, were evaluated. To determine the role of multiple simultaneous risk factors, we evaluated risk profiles using the Essen Stroke Risk Score (ESRS). The ESRS was useful as a predictor of stroke recurrence; we calculated a sum score (0-9 points) based on the following risk factors: 2 points for age $>75$ years and 1 point each for ages 6575 years, hypertension, diabetes mellitus, previous myocardial infarction, other cardiovascular diseases, peripheral arterial disease, current or past ( $<5$ years) smoking, and previous transient ischemic attack (TIA) or ischemic stroke history in addition to the qualifying event [16]

Acute stroke care and optimal medical treatment for secondary stroke prevention were performed based on stroke guidelines [17]. Acute stroke care was performed in the stroke unit, and optimal medical treatment for secondary prevention included modification of blood pressure, lipid profile and glucose intolerance, treatment using antithrombotics and statins, and encouraging patients for better compliance. The primary outcome variable was recurrent stroke of any type. Secondary outcomes included composite cardiovascular events (nonfatal stroke, hospitalization for unstable angina, nonfatal myocardial infarction, and vascular death) and non-vascular death. Recurrent stroke was defined as an acute neurological event that has focal symptoms and signs consistent with a brain lesion and lasting for more than $24 \mathrm{~h}$. Whenever available, a brain CT or MRI was obtained at the time of the recurrent neurological events to confirm the type of recurrent stroke. Posterior circulation ischemic stroke was defined as clinical symptoms correlated with new lesions in the brainstem, cerebellum, or occipital lobe in the vascular territory of vertebrobasilar circulation. Anterior circulation ischemic stroke was defined as clinical symptoms correlated with new lesions in the vascular territory of the anterior cerebral artery, middle cerebral artery, or internal carotid artery. Hemorrhagic stroke was defined as either a parenchymal, subarachnoid, or intraventricular hemorrhage detected on a brain CT or MRI that is associated with the recurrent neurological events. In cases where brain imaging could not be obtained by investigators, if recurrent stroke was confirmed by telephone interview, it was classified as unknown stroke. Deaths directly related to the stroke, myocardial infarction, or other vascular causes within 28 days of the patient first being seen were classified as vascular death. Patient follow-ups were conducted either by a face-to-face interview at an outpatient clinic with a physician or by a telephone interview with a trained nurse at 3 months, 1 year, and every year thereafter. Based on the mean follow-up period used in previous studies of patients who had angioplasty and stenting of the vertebral artery [18], the duration of follow-up was designed to be at least 2 years. This study was approved by the Konkuk University Medical Center Institutional Review Board as a prospective observational study.

\section{MR imaging study}

All MR examinations were performed using a $3 \mathrm{~T}$ MR scanner (Signa HDx; GE Healthcare, Milwaukee, WI, USA) with an 8-channel head coil. T1-, T2-weighted, fluid attenuation inversion recovery, $\mathrm{T} 2 *$ gradient-echo, DWI, and intra- and extracranial MRA were obtained. T1-weighted, T2-weighted, and DWI were obtained during the same imaging session and in the same orientation and slice position: matrix $=512 \times 224$ in T1weighted, $384 \times 384$ in T2-weighted and $128 \times 128$ in 
DWI; field of view $=220 \times 220 \mathrm{~mm}$ in $\mathrm{T} 1$ and $\mathrm{T} 2$ weighted and $240 \times 240 \mathrm{~mm}$ in DWI; section thickness $=5 \mathrm{~mm}$ in $\mathrm{T} 1$ and T2-weighted and $3.5 \mathrm{~mm}$ in DWI; intersection space $=2 \mathrm{~mm}$ in $\mathrm{T} 1$ and $\mathrm{T} 2$-weighted and $0 \mathrm{~mm}$ in DWI; and diffusion gradient strength, two b values $=0$ and $1000 \mathrm{~s} / \mathrm{mm}^{2}$. The MRA consisted of three-dimensional time-of-flight (TOF) sequences with the following parameters: repetition time $(\mathrm{TR})=24 \mathrm{~ms}$; echo time $(\mathrm{TE})=2.6 \mathrm{~ms}$; acquisition $=1$; flip angle $=20^{\circ}$; matrix of $512 \times 512$ using zero-fill interpolation $=384 \times$ 224; effective section thickness $=0.6 \mathrm{~mm}$; and field of view $=220 \times 220 \mathrm{~mm}$. Additionally, CE-MRA was performed for the evaluation of neck vessels using the timeresolved method. A typical injection protocol consisted of a bolus injection of $10 \mathrm{~mL}$ of gadobutrol (Gadovist ${ }^{\circ}$ 1.0; Bayer Schering Pharma AG, Berlin, Germany) at a rate of $2.0 \mathrm{~mL} / \mathrm{s}$, followed by flushing with $35 \mathrm{~mL}$ of saline bolus at the same rate. CE-MRA was acquired with a three-dimensional gradient echo sequence using the time-resolved method (Time-Resolved Imaging of Contrast Kinetics, TRICKS). The acquisition parameters for CE-MRA were as follows: TR/TE $=3 / 1.1 \mathrm{~ms}$; flip angle $=20^{\circ}$; matrix $=320 \times 192$; slice thickness $=2.8 \mathrm{~mm}$; field of view $=320 \times 160 \mathrm{~mm}$; and a bandwidth $=83.33 \mathrm{kHz}$. Three-dimensional volume acquisitions were coronally oriented with section coverage from the aortic arch to the circle of Willis and laterally to both subclavian arteries. Moreover, volume acquisitions were conducted in 13 temporal phases with a temporal resolution time of $2.4 \mathrm{~s}$ and a total scan time of approximately $41 \mathrm{~s}$. Each threedimensional image set was viewed as a coronal maximum-intensity projection.

\section{Other clinical considerations}

To relate the clinical significance of VAO stenosis to the vascular territory of the index stroke, patients were classified into an asymptomatic VAO stenosis group when acute infarcts were observed in the anterior circulation (Figure 1A) or into a symptomatic VAO stenosis group when acute infarcts were observed only in the posterior circulation (Figure 1B). Because symptoms of posterior circulation TIA can be vague, TIA patients who did not have acute ischemic lesions on DWI were excluded from our study. Infarcts in the vascular territory of the posterior inferior cerebellar artery (PICA) and VAO stenosis on the side contralateral to the infarct were also included in the symptomatic VAO stenosis group because PICA branching from basilar artery, anterior inferior cerebellar artery, or contralateral vertebral artery is not a rare variation [19]. However, we could not confirm these variations because we did not perform conventional angiography routinely. The presence of concurrent severe cerebrovascular atherosclerosis, including concurrent severe steno-occlusion of the contralateral vertebral artery, tandem stenosis of the intracranial vertebrobasilar artery or severe concurrent carotid stenosis, and stroke subtype were evaluated as possible risk factors for longterm outcome. Stroke subtypes were classified by two neurologists (JH Lee and HY Kim) according to the modification of the Trial of Org 10172 in Acute Stroke Treatment classification [20]. Because the aim of our study was to investigate the clinical significance of VAO stenosis, VAO stenosis was not considered as the stenosis responsible for the stroke subtype of large artery atherosclerosis. Stroke subtype in some patients who
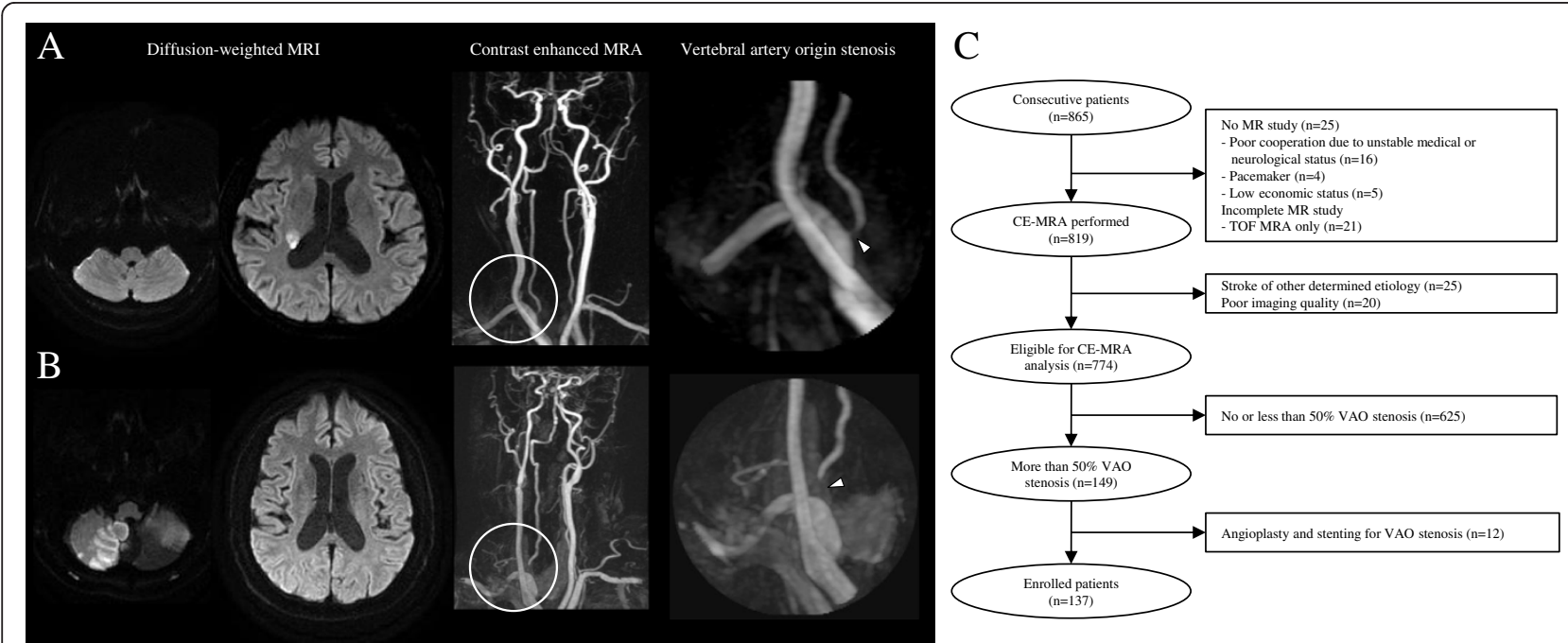

Figure 1 Classification of vertebral artery origin stenosis. Representative cases of asymptomatic stenosis of vertebral artery origin (A) when acute infarcts were observed in the anterior circulation and symptomatic stenosis of vertebral artery origin (B) when acute infarcts were observed only in the posterior circulation. Arrowheads indicate stenosis of vertebral artery origin. (C) Patient inclusion flowchart. 
had symptomatic VAO stenosis was classified as small artery occlusion or cardioembolism instead of two or more causes if they had no other concurrent significant stenosis in the vertebrobasilar system except for VAO stenosis.

\section{Statistical analysis}

The chi square test or Fisher's exact test was used to compare categorical variables, and the $t$ test was used to compare continuous variables, when appropriate. Data were censored at the time of outcome or at the last follow-up assessment. Annual event rates for the study outcomes were calculated as the number of outcomes divided by the patient-years of follow-up. Cardiovascular outcome-free survival was analyzed using Kaplan-Meier survival curves. Univariate and multivariate Cox proportional hazard analyses were used to identify risk factors for recurrent stroke or composite cardiovascular events. A p value of $<0.05$ was considered statistically significant. Statistical analyses were performed using SPSS version 17.0.

\section{Results}

\section{Patient inclusion}

Of the 865 consecutive patients who had acute ischemic stroke, 25 patients (2.9\%) did not undergo an MRI study because of the various following reasons: severe neurological deficits (due to unstable medical or neurological status arising from consciousness disturbance, severe dementia, or mechanical ventilator management, $n=16$ ), use of a pacemaker $(\mathrm{n}=4)$, and low economic status $(\mathrm{n}=5)$ (Figure 1C). Patients who underwent TOF-MRA only, which could not cover the proximal neck vessels, were excluded $(n=21)$. To maintain homogeneity in patient selection, patients who had strokes of other determined etiology were excluded $(n=25)$. In addition, 20 patients who provided poor image quality of their proximal neck vessels were excluded from the final analysis. Among the 774 patients who were eligible for the CEMRA analysis of VAO, 149 (19.3\%) had more than 50\% stenosis of VAO. Additionally, 12 patients who underwent angioplasty and stenting for VAO stenosis within 1 month after stroke-onset were excluded. The mean age was $70.4 \pm 10.3$ years, and other baseline characteristics are listed in Table 1. When comparing the asymptomatic and symptomatic VAO stenosis groups, concurrent vertebrobasilar stenosis was more prevalent in patients with symptomatic VAO stenosis $(21.9 \%$ in the asymptomatic group vs. $38.4 \%$ in the symptomatic group, $\mathrm{p}=0.037)($ Table 1$)$.

Table 1 Baseline characteristics of patients with asymptomatic or symptomatic vertebral artery origin stenosis

\begin{tabular}{|c|c|c|c|c|}
\hline & \multirow{2}{*}{ Total $(n=137)$} & \multicolumn{2}{|c|}{ Vertebral artery origin stenosis } & \multirow{2}{*}{ P-value } \\
\hline & & Asymptomatic $(n=64)$ & Symptomatic $(n=73$ ) & \\
\hline Age, years (mean $\pm S D$ ) & $70.4 \pm 10.3$ & $71.5 \pm 10.8$ & $69.5 \pm 9.9$ & 0.247 \\
\hline Male (n,\%) & $73(53.3)$ & $40(62.5)$ & $33(45.2)$ & 0.043 \\
\hline Hypertension (n,\%) & $96(70.1)$ & $46(71.9)$ & $50(68.5)$ & 0.666 \\
\hline Diabetes $(n, \%)$ & $66(48.2)$ & $30(46.9)$ & $36(49.3)$ & 0.776 \\
\hline Hyperlipidemia (n,\%) & $56(40.9)$ & $28(43.8)$ & $28(38.4)$ & 0.522 \\
\hline Atrial fibrillation (n,\%) & 6. (4.4) & $4(6.3)$ & $2(2.7)$ & 0.316 \\
\hline Smoking (n,\%) & $61(44.5)$ & $29(45.3)$ & $32(43.8)$ & 0.862 \\
\hline Previous stroke history (n,\%) & $34(24.8)$ & $20(31.3)$ & $14(19.2)$ & 0.103 \\
\hline Coronary arterial disease & $27(19.7)$ & $12(18.8)$ & $15(20.5)$ & 0.792 \\
\hline Peripheral arterial disease & $5(3.6)$ & $2(3.1)$ & $3(4.1)$ & 0.759 \\
\hline ESRS (mean $\pm \mathrm{SD}$ ) & $3.2 \pm 1.5$ & $3.3 \pm 1.5$ & $3.1 \pm 1.4$ & 0.339 \\
\hline Concurrent vertebrobasilar stenosis* & $42(30.7)$ & $14(21.9)$ & $28(38.4)$ & 0.037 \\
\hline Concurrent carotid stenosis $^{\dagger}$ & $28(20.4)$ & $16(25.0)$ & $12(16.4)$ & 0.215 \\
\hline Stroke subtype & & & & 0.304 \\
\hline Small artery occlusion & $39(28.5)$ & $21(32.8)$ & $18(24.7)$ & \\
\hline Large artery atherosclerosis & $48(35.0)$ & $24(37.5)$ & $24(32.9)$ & \\
\hline Cardioembolism & $11(8.0)$ & $6(9.4)$ & $5(6.8)$ & \\
\hline Two or more causes & $16(11.7)$ & $4(6.3)$ & $12(16.4)$ & \\
\hline Negative evaluation & $23(16.8)$ & $9(14.1)$ & $14(19.2)$ & \\
\hline
\end{tabular}

*indicates the presence of concurrent severe stenosis or occlusion of the contralateral vertebral artery, or tandem stenosis of intracranial vertebral artery or basilar artery.

${ }^{+}$indicates the concurrent presence of more than $50 \%$ stenosis of at least one carotid artery.

ESRS Essen Stroke Risk Score. 


\section{CE-MRA vs. conventional angiography}

Among 49 VAO stenoses (more than 50\% in CE-MRA) in the 37 patients who underwent both CE-MRA and conventional angiography, 8 (16.3\%) exhibited less than $50 \%$ stenosis in conventional angiography. More than $50 \%$ stenosis was concordant in the remaining $41 \mathrm{VAO}$ stenoses (83.7\%). However, the degree of stenosis measured by CE-MRA was somewhat overestimated compared to the degree measured using conventional angiography.

\section{Performance of the optimal medical treatment}

As indicators of risk factor control, blood pressure, lipid profile, and HbA1c level were measured. Profiles of these indicators were improved at 3 and 12 months of follow-up. Intensity of the optimal medical treatment was also assessed by compliance to taking medication, which included antihypertensive drugs, antithrombotics, or statins. Almost all patients were on antithrombotics (>90\%), and most patients were taking antihypertensive drugs and statins (> 70\%) at 3 and 12 months of followup. Compared to the baseline, follow-up profiles of blood pressure, total cholesterol, LDL cholesterol, and
HbA1c were significantly improved in both groups (Table 2). The performance of optimal medical treatment for risk factor management and control was adequate in both groups.

\section{Stroke recurrence and vascular outcome}

The mean follow-up period was $2.3 \pm 1.2$ years for a total of 309 patient-years. During the follow-up period, there were 7 ischemic strokes (3 in the posterior circulation and 4 in the anterior circulation), 6 hemorrhagic strokes, 2 unknown strokes, and 21 composite cardiovascular outcomes (Table 3). The annual event rates were $0.97 \%$ for posterior circulation ischemic stroke, $1.30 \%$ for anterior circulation ischemic stroke, $1.94 \%$ for hemorrhagic stroke, $4.86 \%$ for all stroke, and $6.80 \%$ for composite cardiovascular outcome (Table 3). The annual event rate of ischemic stroke in the posterior circulation was significantly higher in the symptomatic VAO stenosis group than in the asymptomatic group $(1.88 \%$ vs. $0 \%, \mathrm{p}=0.046)$. Any other individual cardiovascular outcomes were not different between the asymptomatic and symptomatic VAO stenosis groups (Table 3). Using Kaplan-Meier curves, the cumulative risks of posterior circulation

Table 2 Risk factor control and medication assessed at 3 and 12 months of follow-up

\begin{tabular}{|c|c|c|c|c|}
\hline & & Baseline & 3-month follow-up & 12-month follow-up \\
\hline \multirow[t]{10}{*}{ Asymptomatic VAO stenosis $(n=64)$} & Systolic BP (mmHg) & $151.0 \pm 29.3(n=64)$ & $124.5 \pm 15.4(n=51)^{*}$ & $123.4 \pm 13.8(n=42)^{*}$ \\
\hline & Diastolic BP (mmHg) & $84.6 \pm 16.0(n=64)$ & $71.0 \pm 10.1(n=51)^{*}$ & $71.5 \pm 8.8(n=42)^{*}$ \\
\hline & Total cholesterol (mg/dl) & $171.8 \pm 36.7(n=63)$ & & $141.0 \pm 34.7(n=49)^{*}$ \\
\hline & LDL cholesterol (mg/dl) & $107.9 \pm 29.7(n=59)$ & & $80.7 \pm 31.3(n=27)^{*}$ \\
\hline & HDL cholesterol (mg/dl) & $43.3 \pm 10.5(n=63)$ & & $44.8 \pm 11.7(n=48)$ \\
\hline & $\mathrm{HbA1c}(\%)^{\S}$ & $8.0 \pm 2.2(n=30)$ & & $7.0 \pm 1.2(n=23)^{\ddagger}$ \\
\hline & Number of evaluated patients & & 55 & 45 \\
\hline & Antihypertensive drug $(n, \%)$ & & $42(76.4)$ & $37(82.2)$ \\
\hline & $\begin{array}{l}\text { Antiplatelet agent or oral } \\
\text { anticoagulant }(n, \%)\end{array}$ & & $53(96.4)$ & $41(91.1)$ \\
\hline & Statin $(n, \%)$ & & $36(65.5)$ & $33(73.3)$ \\
\hline \multirow[t]{10}{*}{ Symptomatic VAO stenosis $(n=73)$} & Systolic BP (mmHg) & $152.6 \pm 22.6(n=73)$ & $123.3 \pm 16.0(n=57)^{*}$ & $125.6 \pm 14.8(n=49)^{*}$ \\
\hline & Diastolic BP (mmHg) & $82.4 \pm 15.3(n=73)$ & $69.9 \pm 9.7(n=57)^{*}$ & $72.8 \pm 10.6(n=49)^{*}$ \\
\hline & Total cholesterol (mg/dl) & $185.8 \pm 37.3(n=73)$ & & $148.0 \pm 31.7(n=53)^{*}$ \\
\hline & LDL cholesterol (mg/dl) & $112.0 \pm 31.2(n=70)$ & & $81.0 \pm 27.5(n=30)^{*}$ \\
\hline & HDL cholesterol (mg/dl) & $44.9 \pm 10.6(n=73)$ & & $47.1 \pm 17.6(n=52)$ \\
\hline & $\operatorname{HbA1c}(\%)^{\S}$ & $8.3 \pm 1.7(n=35)$ & & $7.3 \pm 0.9(n=22)^{\dagger}$ \\
\hline & Number of evaluated patients & & 59 & 51 \\
\hline & Antihypertensive drug $(n, \%)$ & & $39(66.1)$ & $37(72.5)$ \\
\hline & $\begin{array}{l}\text { Antiplatelet agent or oral } \\
\text { anticoagulant }(n, \%)\end{array}$ & & $58(98.3)$ & 49 (96.1) \\
\hline & Statin $(n, \%)$ & & $45(76.3)$ & $39(76.5)$ \\
\hline
\end{tabular}

Values denote mean \pm SD or numbers with percentages in parentheses. $n$ number of evaluated patients.

Significantly improved compared to profiles of the baseline: ${ }^{*} p<0.001,{ }^{\dagger} p<0.01,{ }^{\ddagger} p<0.05$.

${ }^{\S} \mathrm{HbA} 1 \mathrm{c}$ was measured in patients with diabetes mellitus.

$V A O$ vertebral artery origin. 
Table 3 Incidence and annual event rate of outcomes during follow-up

\begin{tabular}{|c|c|c|c|c|c|c|c|}
\hline & \multicolumn{3}{|c|}{ Incidence, number of patients (\%) } & \multicolumn{3}{|c|}{$\begin{array}{c}\text { Annual event rate, per } 100 \text { patient-years } \\
\text { units }(95 \% \mathrm{Cl})\end{array}$} & \multirow[t]{2}{*}{ P-value } \\
\hline & $\begin{array}{c}\text { Total } \\
(n=137)\end{array}$ & $\begin{array}{l}\text { Asymptomatic } \\
(n=64)\end{array}$ & $\begin{array}{c}\text { Symptomatic } \\
(n=73)\end{array}$ & $\begin{array}{c}\text { Total } \\
(n=137)\end{array}$ & $\begin{array}{l}\text { Asymptomatic } \\
(\mathrm{n}=64)\end{array}$ & $\begin{array}{c}\text { Symptomatic } \\
(n=73)\end{array}$ & \\
\hline All stroke & $15(10.9)$ & $9(14.1)$ & $6(8.2)$ & $4.86(2.93-8.06)$ & $6.02(3.13-11.58)$ & $3.77(1.69-8.38)$ & 0.367 \\
\hline Posterior circulation ischemic stroke & $3(2.2)$ & $0(0)$ & $3(4.1)$ & $0.97(0.31-3.01)$ & 0.00 & $1.88(0.61-5.84)$ & 0.046 \\
\hline Anterior circulation ischemic stroke & $4(2.9)$ & $3(4.7)$ & $1(1.4)$ & $1.30(0.49-3.45)$ & $2.01(0.65-6.23)$ & $0.63(0.09-4.45)$ & 0.277 \\
\hline Hemorrhagic stroke & $6(4.4)$ & $4(6.3)$ & $2(2.7)$ & $1.94(0.87-4.33)$ & $2.68(1.00-7.13)$ & $1.26(0.31-5.02)$ & 0.367 \\
\hline Unknown stroke & $2(1.5)$ & $2(3.1)$ & $0(0)$ & $0.65(0.16-2.59)$ & $1.34(0.33-5.35)$ & 0.00 & 0.088 \\
\hline Unstable angina/myocardial infarction & $4(2.9)$ & $2(3.1)$ & $2(2.7)$ & $1.30(0.49-3.45)$ & $1.34(0.33-5.35)$ & $1.26(0.31-5.02)$ & 0.949 \\
\hline Vascular death & $4(2.9)$ & $2(3.1)$ & $2(2.7)$ & $1.30(0.49-3.45)$ & $1.34(0.33-5.35)$ & $1.26(0.31-5.02)$ & 0.949 \\
\hline Composite cardiovascular outcome & $21(15.3)$ & $11(17.2)$ & $10(13.7)$ & $6.80(4.43-10.43)$ & $7.36(4.08-13.30)$ & $6.28(3.38-11.66)$ & 0.714 \\
\hline Non-vascular death & $14(10.2)$ & $8(12.5)$ & $6(8.2)$ & $4.53(2.69-7.66)$ & $5.36(2.68-10.71)$ & $3.77(1.69-8.38)$ & 0.512 \\
\hline
\end{tabular}

*p-value was calculated from annual event rates of the asymptomatic and symptomatic groups.

ischemic stroke, all stroke, and composite cardiovascular outcome according to the asymptomatic and symptomatic VAO stenosis groups were plotted (Figure 2).

Cox proportional hazard analyses indicated that individual vascular risk factors, ESRS, symptomatic stenosis of VAO, and concurrent stenosis of other vertebrobasilar or carotid circulation were not associated with any type of stroke outcome, including posterior circulation ischemic stroke (data not shown). The presence of peripheral arterial disease and ESRS were associated with the composite cardiovascular outcome in the univariate Cox proportional hazard analyses (Table 4). Multivariate Cox proportional hazard analyses revealed that the significant association with peripheral arterial disease disappeared in model 1, and only ESRS was associated with composite cardiovascular outcome in model 2 (Table 4). The hazard ratio, per one point increase of the ESRS for the composite cardiovascular outcome, was 1.46 (95\% CI, 1.02-2.08, $\mathrm{p}=0.036$ ).

\section{Discussion}

\section{CE-MRA for the evaluation of VAO}

Among various noninvasive techniques, recent studies have shown that CE-MRA may be the most accurate technique for evaluating stenosis of the carotid artery [21] or vertebral artery [22,23]. Compared to conventional angiography, CE-MRA may have some limitations in assessing the exact degree of stenosis at the VAO [24]. However, recent studies have shown reliable diagnostic accuracy between conventional angiography and CE-MRA, demonstrating a sensitivity of $88 \%$ and a specificity of $98 \%$ for more than $50 \%$ stenosis in the vertebrobasilar system
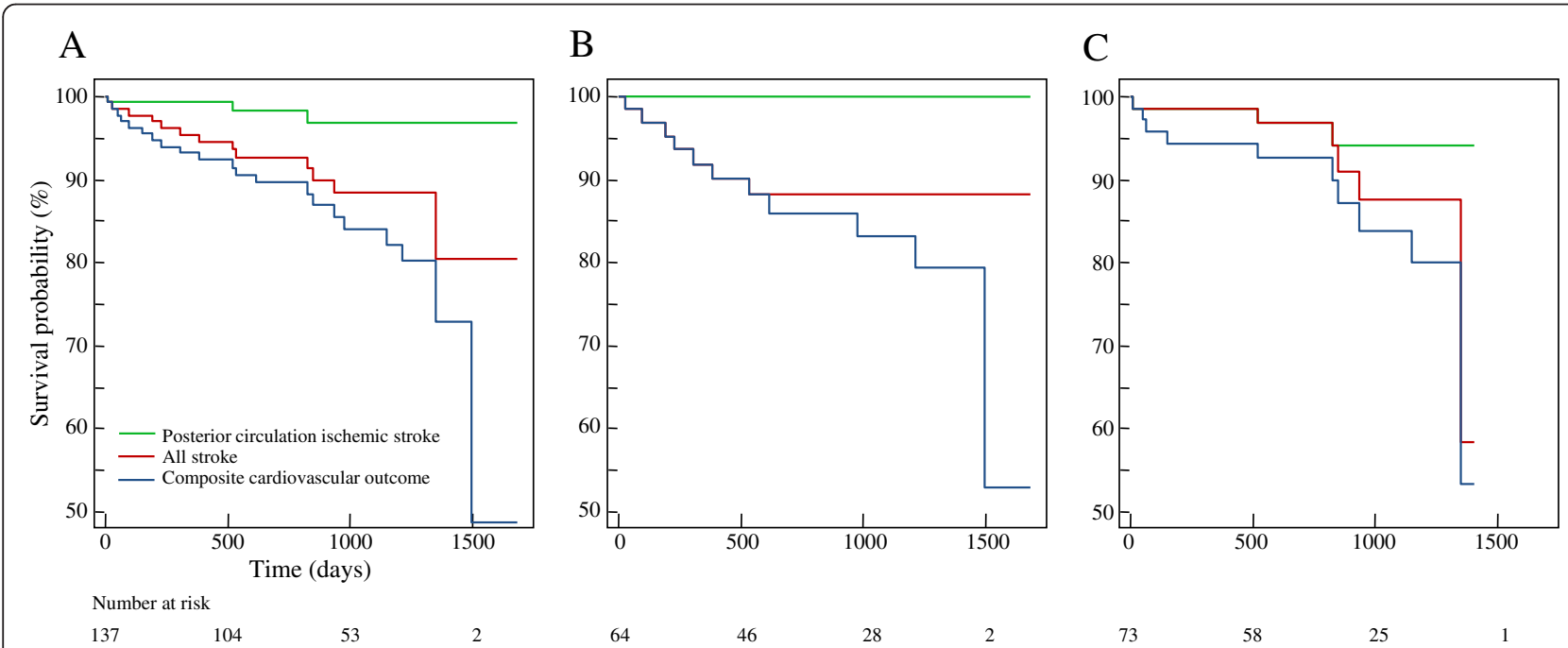

Figure 2 Long-term outcome of vertebral artery origin stenosis. The cumulative risk of posterior circulation ischemic stroke, all stroke, and the composite cardiovascular outcome of all patients (A), the asymptomatic stenosis group (B), and the symptomatic stenosis group (C) were plotted using Kaplan-Meier curves. 
Table 4 Predictors of composite cardiovascular outcome in the univariate and multivariate Cox proportional hazard analyses

\begin{tabular}{|c|c|c|c|c|c|c|}
\hline & \multirow{2}{*}{\multicolumn{2}{|c|}{ Univariate analysis }} & \multicolumn{4}{|c|}{ Multivariate analysis } \\
\hline & & & \multicolumn{2}{|l|}{ Model 1} & \multicolumn{2}{|l|}{ Model 2} \\
\hline & Hazard ratio $(95 \% \mathrm{Cl})$ & P-value & Hazard ratio $(95 \% \mathrm{Cl})$ & P-value & Hazard ratio $(95 \% \mathrm{Cl})$ & P-value \\
\hline Age (per 1 year) & $1.01(0.97-1.05)$ & 0.720 & $1.00(0.95-1.06)$ & 0.893 & - & \\
\hline Sex, male & $0.95(0.40-2.23)$ & 0.901 & $0.77(0.22-2.70)$ & 0.678 & $0.83(0.34-2.05)$ & 0.685 \\
\hline Hypertension & $2.27(0.66-7.75)$ & 0.181 & $2.10(0.56-7.82)$ & 0.271 & - & \\
\hline Diabetes & $2.21(0.88-5.55)$ & 0.082 & $1.89(0.70-5.11)$ & 0.208 & - & \\
\hline Hyperlipidemia & $1.32(0.56-3.14)$ & 0.522 & $1.31(0.52-3.33)$ & 0.570 & $1.54(0.64-3.72)$ & 0.340 \\
\hline Atrial fibrillation & $1.39(0.31-6.32)$ & 0.669 & $1.56(0.24-10.24)$ & 0.642 & $1.19(0.23-6.10)$ & 0.837 \\
\hline Smoking & $1.19(0.49-2.90)$ & 0.697 & $1.52(0.40-5.77)$ & 0.538 & - & \\
\hline Previous stroke history & $1.33(0.51-3.44)$ & 0.557 & $1.40(0.51-3.82)$ & 0.516 & - & \\
\hline Coronary arterial disease & $1.26(0.45-3.59)$ & 0.661 & $0.99(0.31-3.17)$ & 0.984 & - & \\
\hline Peripheral arterial disease & $4.99(1.13-22.0)$ & 0.034 & $8.29(0.92-74.52)$ & 0.059 & - & \\
\hline ESRS (per 1) & $1.38(1.01-1.88)$ & 0.045 & - & & $1.46(1.02-2.08)$ & 0.036 \\
\hline Symptomatic stenosis of vertebral artery origin* & $0.99(0.41-2.39)$ & 0.978 & $1.09(0.40-2.95)$ & 0.869 & $1.04(0.40-2.74)$ & 0.935 \\
\hline Concurrent vertebrobasilar stenosis $^{\ddagger}$ & $1.61(0.67-3.90)$ & 0.287 & $1.12(0.39-3.21)$ & 0.837 & $1.35(0.52-3.55)$ & 0.540 \\
\hline Concurrent carotid stenosis ${ }^{\ddagger}$ & $0.90(0.30-2.68)$ & 0.848 & $0.41(0.89-1.91)$ & 0.256 & $0.65(0.21-2.06)$ & 0.467 \\
\hline
\end{tabular}

and a sensitivity of $100 \%$ and a specificity of $85 \%$ for more than $50 \%$ stenosis at the VAO [25,26]. In our study, the concordance rate for selected patients who underwent both CE-MRA and conventional angiography was acceptable as high as $83.7 \%$. Considering the relative invasiveness of conventional angiography, CE-MRA may be a safe and reliable alternative for the evaluation of VAO in patients with acute ischemic stroke.

\section{Prevalence of VAO stenosis}

The prevalence of VAO stenosis is variable according to different study populations and ranges from 7.6 to $44.4 \%$ $[3,5,24,27]$. In a hospital-based cohort of patients with atherosclerotic arterial disease who were enrolled in the Second Manifestation of ARTerial disease (SMART) study, 282 patients $(7.6 \%)$ presented asymptomatic VAO stenosis of more than $50 \%$ among a total of 3717 patients [5]. The presence of asymptomatic VAO stenosis was more frequent in the subgroup that had previous ischemic stroke history compared to the subgroup that did not $(22 / 225,9.8 \%$ vs. $260 / 3492,7.4 \%)$ [5]. In a prospective population-based study nested within the Oxford Vascular Study, 16 patients (11.3\%) had more than $50 \%$ stenosis at or near the VAO (9 at the vertebral origin and 7 near the origin) among a total of 141 patients who had posterior circulation vascular events [24].
Among 407 New England Medical Center Posterior Circulation registry patients, 131 patients $(32.1 \%)$ presented more than $50 \%$ stenosis of the VAO [3]. In a nonconsecutive cohort consisted of various patients who had TIA or stroke and some asymptomatic patients, the prevalence of more than $50 \%$ stenosis of the proximal vertebral artery was as high as $27.3 \%(33 / 121)$ in patients who had anterior circulation infarcts and 44.4\% (32/72) in patients who had posterior circulation infarcts [27]. Taken together, VAO stenosis may be more prevalent in patients who have had an ischemic stroke than in patients who have had other atherosclerotic arterial diseases [5]. Among patients with ischemic stroke, VAO stenosis may be more frequently observed in patients who have had strokes in the posterior circulation compared to patients who have had strokes in the anterior circulation [27]. The prevalence of VAO stenosis (19.3\%, $149 / 774$ ) in our hospital-based cohort of patients with acute ischemic stroke was comparable to those reported in previous studies. In our study, the prevalence of VAO stenosis was higher in patients with posterior circulation ischemic stroke than that in patients with anterior circulation ischemic stroke $(31.7 \%, 85 / 268$ posterior circulation ischemic stroke vs.12.6\%, 64/506 anterior or simultaneous anterior and posterior circulation ischemic stroke, $\mathrm{p}<0.001)$. In particular, the prevalence of VAO 
stenosis in the subgroup of patients with posterior circulation ischemic stroke in our study cohort (31.7\%) was comparable to those from previous cohort studies consisted of patients who had posterior circulation stroke $\mathrm{s}$ (i.e., 11.3-44.4\%) [3,24,27]. The higher incidence of VAO stenosis in patients who had ischemic strokes in the posterior circulation compared to the anterior circulation may suggest VAO stenosis as a possible cause of ischemic stroke in the posterior circulation.

\section{Low risk of posterior circulation ischemic stroke in patients with VAO stenosis}

With the development of medical treatments, therapeutic strategies for patients who have asymptomatic carotid stenosis have recently been reevaluated [28,29]. Owing to the low recurrence rate of stroke under current optimal medical treatment, medical treatment may be more cost-effective than carotid endarterectomy or angioplasty and stenting for stroke prevention [28]. Given that VAO stenosis is a counterpart of proximal carotid stenosis in the posterior circulation, it may be interesting to compare our outcome data of the asymptomatic VAO stenosis group to data from a recent study of patients with asymptomatic carotid stenosis under the best medical treatment [29]. In this population-based study of patients who had TIA or stroke, the annual rate of ipsilateral vascular events remained quite low in 101 patients with asymptomatic carotid stenosis of more than 50\% undergoing intensive medical treatment (i.e., ipsilateral $\mathrm{TIA}=1.78 \%$ and ipsilateral stroke $=0.34 \%$ ) [29]. The pattern of stroke recurrence in this asymptomatic carotid stenosis study showed trends similar to the asymptomatic VAO stenosis group of our study. The annual rate of stroke recurrence in the vascular territory of asymptomatic stenosis was very low in both studies (i.e., posterior circulation ischemic stroke in our asymptomatic VAO stenosis group $=0 \%$ vs. ipsilateral anterior circulation stroke in the asymptomatic carotid study= $0.34 \%)$. Most recurrent strokes occurred in other vascular territories unrelated to asymptomatic stenosis (i.e., all stroke in our asymptomatic VAO stenosis group $=6.02 \%$ vs. other territory strokes in the asymptomatic carotid stenosis study $=8.32 \%$ ). In our study, recurrent ischemic stroke in the posterior circulation occurred only in the symptomatic VAO stenosis group, and the annual rate of posterior circulation ischemic stroke was significantly higher in the symptomatic VAO stenosis group (i.e., $0 \%$ in the asymptomatic group vs. $1.88 \%$ in the symptomatic group, $\mathrm{p}=0.046$ ). A higher prevalence of concurrent vertebrobasilar stenosis in the symptomatic VAO stenosis group could have contributed to the worse outcome of the posterior circulation ischemic stroke.

We compared outcomes and variates of patients enrolled in our study to a previously published study that used the same stroke registry in a similar period [14]. Patients enrolled in the previous study had acute lacunar infarcts and were comparable to acute ischemic stroke patients who did not have intra- or extra-cranial artery stenosis. The baseline characteristics of patients with acute lacunar infarcts were not different from those of VAO stenosis except for age (Table 5). ESRS was slightly higher in the VAO stenosis group, but this increase was not statistically significant. The annual event rates of outcomes were not different (Table 6). Considering the most favorable outcomes were in patients with lacunar infarcts among stroke subtypes [30], outcomes of the VAO stenosis group comparable to those of lacunar infarcts may support a hypothesis that there will be a generally favorable outcome in patients with VAO stenosis.

Considering the potential risk of procedure-related stroke or death in patients treated with angioplasty and stenting, the low absolute risk of posterior circulation ischemic stroke in patients with asymptomatic VAO stenosis who are on the best medical treatment may not warrant invasive treatment of asymptomatic VAO stenosis [5]. Because the majority of patients with asymptomatic VAO stenosis die from vascular causes other than posterior circulation ischemic stroke, therapeutic strategies should be focused on reducing the total vascular risk [5]. In a long-term follow-up study of 96 patients who had mainly VAO stenosis, recurrence of posterior circulation ischemic stroke itself was low (i.e., 2\% during an average follow-up of 4.6 years) [10]. Instead, the risk of cardiovascular complications was higher in these patients than in the matched normal population [10]. In our study, individual vascular risk factors, symptomatic

Table 5 Baseline characteristics of patients with vertebral artery origin stenosis or acute lacunar infarcts

\begin{tabular}{lccc}
\hline & $\begin{array}{c}\text { Vertebral artery } \\
\text { origin stenosis } \\
(\mathbf{n}=\mathbf{1 3 7})\end{array}$ & $\begin{array}{c}\text { Acute } \\
\text { lacunar infarcts } \\
(\mathbf{n}=\mathbf{1 7 1})\end{array}$ & P-value \\
\hline Age, years (mean \pm SD) & $70.4 \pm 10.3$ & $63.8 \pm 11.5$ & $<0.001$ \\
Male (n,\%) & $73(53.3)$ & $97(56.7)$ & 0.566 \\
Hypertension (n,\%) & $96(70.1)$ & $119(69.6)$ & 1.000 \\
Diabetes (n,\%) & $66(48.2)$ & $75(43.9)$ & 0.491 \\
Hyperlipidemia (n,\%) & $56(40.9)$ & $57(33.3)$ & 0.191 \\
Atrial fibrillation (n,\%) & $6 .(4.4)$ & $5(2.9)$ & 0.547 \\
Smoking (n,\%) & $61(44.5)$ & $90(52.6)$ & 0.170 \\
Previous stroke history (n,\%) & $34(24.8)$ & $41(24.0)$ & 0.894 \\
Coronary arterial disease & $27(19.7)$ & $41(24.4)$ & 0.337 \\
Peripheral arterial disease & $5(3.6)$ & $6(3.6)$ & 1.000 \\
ESRS (mean \pm SD) & $3.2 \pm 1.5$ & $2.9 \pm 1.5$ & 0.126 \\
ESRS $>3$ & $52(38.0)$ & $58(33.9)$ & 0.475 \\
\hline
\end{tabular}

ESRS Essen Stroke Risk Score.

Published data of patients who had acute lacunar infarcts were used for the comparison; data used with personal permission [14]. 
Table 6 Annual event rate of outcomes during follow-up of patients with vertebral artery origin stenosis or acute lacunar infarcts

\begin{tabular}{lccc}
\hline & \multicolumn{2}{c}{ Annual event rate, per $\mathbf{1 0 0}$ patient-years units $(\mathbf{9 5 \%} \mathrm{Cl})$} & $\mathbf{P}$-value \\
\cline { 2 - 3 } & Vertebral artery origin stenosis $(\mathbf{n}=\mathbf{1 3 7})$ & Acute lacunar infarcts $(\mathbf{n}=\mathbf{1 6 9 )}$ & $3.38(1.41-8.13)$ \\
\hline All stroke & $4.86(2.93-8.06)$ & $2.71(1.02-7.21)$ & 0.486 \\
Unstable angina/myocardial infarction & $1.30(0.49-3.45)$ & $1.35(0.34-5.41)$ & 0.293 \\
Vascular death & $1.30(0.49-3.45)$ & $6.77(3.64-12.58)$ & 0.960 \\
Composite cardiovascular outcome & $6.80(4.43-10.43)$ & 0.990 \\
\hline
\end{tabular}

Published data of patients who had acute lacunar infarcts were used for the comparison; data used with personal permission [14].

stenosis of VAO, or concurrent stenosis of other vertebrobasilar or carotid circulation were not associated with individual outcomes, including any type of stroke. Instead, ESRS, the sum of underlying multiple vascular risk factors, was associated with composite cardiovascular outcome.

Taken together, these results indicate that VAO stenosis itself may not be a specific risk factor for posterior circulation ischemic stroke, especially when it is asymptomatic. However, patients who have higher ESRS need more clinical attention because of the potential of more frequent future cardiovascular events.

\section{Angioplasty and stenting for VAO stenosis}

A recent systematic review reported favorable outcomes of angioplasty and stenting in patients who had mostly symptomatic extracranial vertebral artery stenosis: i.e., posterior circulation ischemic stroke (1.3\%) and TIA (6.5\%) during the average follow-up period of 21 months [18]. Despite the limitation of direct comparison to the results of the systematic review, an annual event rate of $0.97 \%$ for posterior circulation ischemic stroke in our patients who had optimal medical treatment (i.e., $0 \%$ in the asymptomatic group and $1.88 \%$ in the symptomatic group) does not seem to be inferior to the historical outcome data of patients who underwent angioplasty and stenting. Although angioplasty and stenting for VAO stenosis are technically safe and feasible, data on the natural history of patients who have extracranial vertebral artery stenosis are still limited. Moreover, little is known about the most cost-effective treatment option. A randomized trial that compares medical treatment to medical treatment plus additional endovascular treatment is necessary in the future. Because individual VAO stenosis may have different clinical significance according to whether the lesion is symptomatic or not, future study subjects should be clearly defined regarding their symptomatic status for VAO stenosis.

Despite the controversial results of previous studies that focused on VAO stenosis as a possible embolic source for ischemic stroke in the posterior circulation $[8,31]$, some patients with $\mathrm{VAO}$ stenosis may be at a truly higher risk for posterior circulation stroke $[9,12]$.
Although there has been no clear consensus on the management of VAO stenosis, the presence of concurrent severe cerebrovascular atherosclerosis has been considered a reasonable indication for angioplasty and stenting under the potential high risk of recurrent ischemic stroke in the posterior circulation $[8,18,32]$. In our study cohort, the 12 patients who underwent angioplasty and stenting within 1 month after stroke-onset were excluded from our study. A decision for the angioplasty and stenting was based on the individual case-by-case situations: as procedures for acute intra-arterial thrombolysis in 3 patients and concurrent vertebrobasilar and/ or carotid atherosclerosis in 9 patients. All of those patients had acute ischemic stroke in the posterior circulation, and they were potentially at high risk for recurrent stroke. Exclusion of those patients from the symptomatic VAO stenosis group might have biased our results. Therefore, type II error may have occurred in our study because of the small number of subjects, very low recurrence rate of posterior circulation ischemic stroke, and the exclusion of some high risk patients. However, the presence of concurrent vertebrobasilar or carotid atherosclerosis was not associated with the recurrence of posterior circulation ischemic stroke. One-sided preferences for angioplasty and stenting in patients who have VAO stenosis, simply because they have concurrent vertebrobasilar or carotid arterial stenosis, may need more careful consideration.

\section{Limitations of our study}

The number of enrolled patients was relatively small in our study. Although the enrolled patients were prospectively collected from the stroke registry with predefined outcome follow-ups, the data were analyzed retrospectively. Therefore, some degree of bias is inevitable.

Recently, clinical concepts of optimal medical treatment have been introduced: so-called best medical treatment [29], intensive contemporary medical therapy [33], medical intervention [28], aggressive medical management [34], and others. However, specifically detailed guidelines for optimal medical treatment in a real clinical practice have not yet been established. Although we tried to provide optimal medical treatment for all 
patients according to the guidelines of secondary stroke prevention, our medical treatment may have not been optimal enough in some cases.

\section{Conclusions}

Long-term outcome of more than 50\% stenosis of VAO in patients who had acute ischemic stroke was generally favorable with optimal medical treatment. Additionally, ESRS was a predictor for the composite cardiovascular outcome. The clinical significance of VAO stenosis may be different according to the symptomatic status of VAO stenosis. Asymptomatic VAO stenosis may not be a specific risk factor for recurrent ischemic stroke in the posterior circulation. However, when VAO stenosis is observed in patients who had concurrent ischemic strokes in the posterior circulation (i.e., symptomatic VAO stenosis), more clinical attention on VAO stenosis may be needed because this condition could be a potential source of recurrent stroke.

\section{Competing interests}

The authors declare that they have no competing interests.

\begin{abstract}
Authors' contributions
HY Kim designed the study, interpreted the data, performed the statistical analyses, and drafted the manuscript. YJ Kim and JH Lee participated in the design of study, statistical analyses, and patient enrollment and helped to draft the manuscript. JW Choi, HG Roh, and YI Chun participated in patient enrollment. JS Lee helped to perform the statistical analyses. All authors read and approved the final manuscript.
\end{abstract}

\section{Funding}

This work was supported by Konkuk University Medical Center Research Grant 2012.

\begin{abstract}
Author details
${ }^{1}$ Department of Neurology, Konkuk University Medical Center, Research Institute of Medical Science, Konkuk University School of Medicine, Seoul, Republic of Korea. ${ }^{2}$ Department of Radiology, Konkuk University Medical Center, Research Institute of Medical Science, Konkuk University School of Medicine, Seoul, Republic of Korea. ${ }^{3}$ Department of Neurosurgery, Konkuk University Medical Center, Research Institute of Medical Science, Konkuk University School of Medicine, Seoul, Republic of Korea. ${ }^{4}$ Department of Biostatistics, College of Medicine, Korea University, Seoul, Republic of Korea.
\end{abstract}

Received: 24 June 2013 Accepted: 5 November 2013

Published: 11 November 2013

\section{References}

1. Hong KS, Yegiaian S, Lee M, Lee J, Saver JL: Declining stroke and vascular event recurrence rates in secondary prevention trials over the past 50 years and consequences for current trial design. Circulation 2011 , 123:2111-2119.

2. Bogousslavsky J, Van Melle G, Regli F: The Lausanne stroke registry: analysis of 1,000 consecutive patients with first stroke. Stroke 1988, 19:1083-1092.

3. Caplan LR, Wityk RJ, Glass TA, Tapia J, Pazdera L, Chang HM, Teal P, Dashe JF, Chaves CJ, Breen JC, et al: New England medical center posterior circulation registry. Ann Neurol 2004, 56:389-398.

4. Hass WK, Fields WS, North RR, Kircheff II, Chase NE, Bauer RB: Joint study of extracranial arterial occlusion. II. Arteriography, techniques, sites, and complications. JAMA 1968, 203:961-968.

5. Compter A, van der Worp HB, Algra A, Kappelle LJ: Prevalence and prognosis of asymptomatic vertebral artery origin stenosis in patients with clinically manifest arterial disease. Stroke 2011, 42:2795-2800.
6. Caplan LR, Amarenco P, Rosengart A, Lafranchise EF, Teal PA, Belkin M, DeWitt LD, Pessin MS: Embolism from vertebral artery origin occlusive disease. Neurology 1992, 42:1505-1512.

7. Koroshetz WJ, Ropper AH: Artery-to-artery embolism causing stroke in the posterior circulation. Neurology 1987, 37:292-295.

8. Al-Ali F, Barrow T, Duan L, Jefferson A, Louis S, Luke K, Major K, Smoker S, Walker $S$, Yacobozzi M: Vertebral artery ostium atherosclerotic plaque as a potential source of posterior circulation ischemic stroke: result from borgess medical center vertebral artery ostium stenting registry. Stroke 2011, 42:2544-2549.

9. Gulli G, Khan S, Markus HS: Vertebrobasilar stenosis predicts high early recurrent stroke risk in posterior circulation stroke and TIA. Stroke 2009, 40:2732-2737.

10. Moufarrij NA, Little JR, Furlan AJ, Williams G, Marzewski DJ: Vertebral artery stenosis: long-term follow-up. Stroke 1984, 15:260-263.

11. Moufarrij NA, Little JR, Furlan AJ, Leatherman JR, Williams GW: Basilar and distal vertebral artery stenosis: long-term follow-up. Stroke 1986, 17:938-942.

12. Gulli G, Marquardt L, Rothwell PM, Markus HS: Stroke risk after posterior circulation stroke/transient ischemic attack and its relationship to site of vertebrobasilar stenosis: pooled data analysis from prospective studies. Stroke 2013, 44:598-604.

13. Cho HJ, Lee JH, Kim YJ, Moon Y, Ko SM, Kim HY: Comprehensive evaluation of coronary artery disease and aortic atherosclerosis in acute ischemic stroke patients: usefulness based on Framingham risk score and stroke subtype. Cerebrovasc Dis 2011, 31:592-600.

14. Lee JH, Kim YJ, Moon Y, Cho HJ, Kim HY: Acute simultaneous multiple lacunar Infarcts: a severe disease entity in small artery disease. Eur Neurol 2012, 67:303-311.

15. North American Symptomatic Carotid Endarterectomy Trial Collaborators: Beneficial effect of carotid endarterectomy in symptomatic patients with high-grade carotid stenosis. N Engl J Med 1991, 325:445-453.

16. Weimar C, Diener HC, Alberts MJ, Steg PG, Bhatt DL, Wilson PW, Mas JL, Rother J: The Essen stroke risk score predicts recurrent cardiovascular events: a validation within the REduction of atherothrombosis for continued health (REACH) registry. Stroke 2009, 40:350-354.

17. The writing group of clinical practice guideline for stroke: Clinical Practice Guideline for Stroke. 1st edition. Seoul: Clinical Research Center for Stroke; 2009 [http://stroke-crc.or.kr/CPGs_for_Stroke_(English)_revision_20130730.pdf].

18. Stayman AN, Nogueira RG, Gupta R: A systematic review of stenting and angioplasty of symptomatic extracranial vertebral artery stenosis. Stroke 2011, 42:2212-2216.

19. Macchi V, Porzionato A, Parenti A, De Caro R: The course of the posterior inferior cerebellar artery may be related to its level of origin. Surg Radio Anat 2004, 26:60-65.

20. Adams HP Jr, Bendixen BH, Kappelle LJ, Biller J, Love BB, Gordon DL, Marsh EE 3rd: Classification of subtype of acute ischemic stroke. Definitions for use in a multicenter clinical trial. TOAST. Trial of Org 10172 in acute stroke treatment. Stroke 1993, 24:35-41.

21. Chappell FM, Wardlaw JM, Young GR, Gillard JH, Roditi GH, Yip B, Pell JP, Rothwell PM, Brown MM, Gough MJ, et al: Carotid artery stenosis: accuracy of noninvasive tests-individual patient data meta-analysis. Radiology 2009, 251:493-502.

22. Khan S, Rich P, Clifton A, Markus HS: Noninvasive detection of vertebral artery stenosis: a comparison of contrast-enhanced MR angiography, CT angiography, and ultrasound. Stroke 2009, 40:3499-3503.

23. Khan S, Cloud GC, Kerry S, Markus HS: Imaging of vertebral artery stenosis: a systematic review. J Neurol Neurosurg Psychiatry 2007, 78:1218-1225.

24. Marquardt L, Kuker W, Chandratheva A, Geraghty O, Rothwell PM: Incidence and prognosis of $>$ or $=50 \%$ symptomatic vertebral or basilar artery stenosis: prospective population-based study. Brain 2009, 132(Pt 4):982-988

25. Randoux B, Marro B, Koskas F, Chiras J, Dormont D, Marsault C: Proximal great vessels of aortic arch: comparison of three-dimensional gadolinium-enhanced MR angiography and digital subtraction angiography. Radiology 2003, 229:697-702

26. Yang CW, Carr JC, Futterer SF, Morasch MD, Yang BP, Shors SM, Finn JP: Contrast-enhanced MR angiography of the carotid and vertebrobasilar circulations. AJNR Am J Neuroradio/ 2005, 26:2095-2101.

27. Kim SH, Lee JS, Kwon OK, Han MK, Kim JH: Prevalence study of proximal vertebral artery stenosis using high-resolution contrast-enhanced magnetic resonance angiography. Acta Radiol 2005, 46:314-321.

28. Abbott AL: Medical (nonsurgical) intervention alone is now best for prevention of stroke associated with asymptomatic severe carotid 
stenosis: results of a systematic review and analysis. Stroke 2009, 40:e573-e583.

29. Marquardt L, Geraghty OC, Mehta Z, Rothwell PM: Low risk of ipsilateral stroke in patients with asymptomatic carotid stenosis on best medical treatment: a prospective, population-based study. Stroke 2010, 41:e11-e17.

30. Hong KS, Bang OY, Kang DW, Yu KH, Bae HJ, Lee JS, Heo JH, Kwon SU, Oh CW, Lee BC, et al: Stroke statistics in Korea: part I. Epidemiology and risk factors: a report from the Korean stroke society and clinical research center for stroke. J Stroke 2013, 15:2-20.

31. Hwang J, Kim SJ, Hong JM, Bang OY, Chung CS, Lee KH, Kim GM: Microembolic signals in acute posterior circulation cerebral ischemia: sources and consequences. Stroke 2012, 43:747-752.

32. Taylor RA, Siddiq F, Memon MZ, Qureshi Al, Vazquez G, Hayakawa M, Chaloupka JC: Vertebral artery ostial stent placement for atherosclerotic stenosis in 72 consecutive patients: clinical outcomes and follow-up results. Neuroradiology 2009, 51:531-539.

33. Brott TG, Halperin JL, Abbara S, Bacharach JM, Barr JD, Bush RL, Cates CU, Creager MA, Fowler SB, Friday G, et al: 2011 ASA/ACCF/AHA/AANN/AANS/ ACR/ASNR/CNS/SAIP/SCAI/SIR/SNIS/SVM/SVS guideline on the management of patients with extracranial carotid and vertebral artery disease. Circulation 2011, 124:e54-e130.

34. Chimowitz MI, Lynn MJ, Derdeyn CP, Turan TN, Fiorella D, Lane BF, Janis LS, Lutsep HL, Barnwell SL, Waters MF, et al: Stenting versus aggressive medical therapy for intracranial arterial stenosis. N Engl J Med 2011, 365:993-1003

doi:10.1186/1471-2377-13-171

Cite this article as: Kim et al.: Long-term outcome of vertebral artery origin stenosis in patients with acute ischemic stroke. BMC Neurology 2013 13:171.

\section{Submit your next manuscript to BioMed Central and take full advantage of:}

- Convenient online submission

- Thorough peer review

- No space constraints or color figure charges

- Immediate publication on acceptance

- Inclusion in PubMed, CAS, Scopus and Google Scholar

- Research which is freely available for redistribution 